\title{
An Explanation of Computation - Collective Electrodynamics in Blobs of Carbon Nanotubes
}

\author{
Dragana Laketić, Gunnar Tufte, Odd Rune Lykkebø and Stefano Nichele \\ Department for Computer and Information Science \\ Norwegian University of Science and Technology \\ Trondheim, Norway \\ \{draganal,gunnart,lykkebo,nichele\}@idi.ntnu.no
}

\begin{abstract}
In this short paper an attempt is made to explain computations in nanomaterials under the Evolution-In-Materio scenario. Computations performed by the material are considered within the framework of system theory as introduced in classical cybernetics. Three conceptual domains of computations are identified which are related to different hierarchical levels. Further, a deeper look is taken into the physics of one material in particular since it is extensively used in our experiments. It is revealed that the physics of the investigated nanocomposites, which is the basis for computations, can be explained as a collective property of the wave functions which describe electrons moving within the material. The explanation of computations given in this paper is found valuable for identifying further research directions. The first direction is towards how to manipulate nanomaterials for achieving computations at measurable levels. Manipulation at quantum level is suggested as a possible domain of material manipulation. Secondly, another research direction is identified towards using parameters, environmental parameters for example, for computing.
\end{abstract}

\section{Keywords}

Evolution-In-Materio, carbon nanotubes, system theory, collective electrodynamics

\section{INTRODUCTION}

In our work the notion of computation is mentioned too often to be set aside and not addressed in a greater detail. Computations are physical [6]. Computations happen as a result of a change in a computing substrate which produces a response to some excitation. The excitation and the response are related to one another based on the changes the computing substrate undergoes. This change is purely dependent on the physical properties of the substrate, the state of the substrate when excitation arrives and the type of excitation.
The computing machine usually entails the computing substrate which is endowed with additional entities in order to enable communication with it and suitable transformations of excitation and response signals in order to provide users with an intelligible solution to some computational task. Therefore, it is often referred to as a computing system. When a computing machine is mentioned, the first thing that comes to mind is a digital computer built in semiconductor technology. The computing substrate is therefore a carefully designed microelectronic system where computations happen due to the changes in the substrate made of semiconductors, predominantly silicon.

On the other hand, a biological system computes in a different way. Computations are performed in parallel by myriads of its molecules, inputs to the system have various qualities, the state of the system is dependent on so many variables that it is almost impossible to number them all. The amazing properties of such computing systems, arisen from evolutionary processes, have been a subject of investigation for centuries. The last century in particular saw the rise in such interest among the members of the cybernetics movement [13]. Different computing substrates were examined within system theory framework and valuable findings achieved in a sense that the principles inherent in biological systems like adaptation, ability to solve complex problems in parallel etc. can be successfully used in machines.

The investigation of similar ideas has recently begun within a NASCENCE (NAnoSCale Engineering for Novel Computations using Evolution) project [2,22]. Novel substrates are investigated for computational properties, representatives of nanomaterials and nanoparticles whose nanoscale properties are used for computations. Beside novel computing substrates, NASCENCE investigates novel computing paradigms where computations are analogue in nature and achieved as a result of evolutionary changes undergone by the computing substrate. This approach is termed EvolutionIn-Materio (EIM) [20].

The computing system under investigation is set within a framework of classical cybernetics as in the work of Ashby [1]. Electrical properties used for computations are recognised to arise as an emergent property of the material when put under certain stimulation. At the very bottom of the hierarchical levels of the physicality of the computing substrate, it is the wave functions of the electrons that are manifested at higher levels as an electromagnetic field which is the main physical phenomenon manipulated for computations in EIM. Therefore, the manipulation at this fundamental level may prove suitable for achieving computations at a higher 


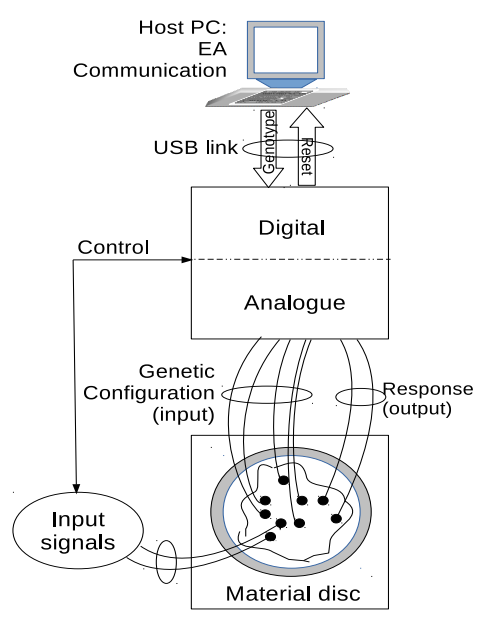

Figure 1: Block diagram of experimental setup for EIM.

and measurable level.

The paper is organised as follows. Section 2 introduces EIM and, as a special case, the materials based on carbon nanotubes (CNT) which are used in our experiments. Section 3 presents the system theory framework and sets the EIM system investigated in NASCENCE within such framework. Section 4 relates identified conceptual domains of computations to different hierarchical levels of the electromagnetic reality in the investigated material. Finally, conclusion and guidelines for further work are presented in Section 5 .

\section{EVOLUTION-IN-MATERIO}

EIM $[10,21]$ is based on two premises. The first is inspired by the work of Gordon Pask and his experiments with self-assembling filaments in ferrous sulphate under stimulation by electrical signals [24]. The resulting electrochemical system exhibited a tone discriminator functionality. The second premise is based on the bottom up approach to the search for a solution to some computational task. It includes some form of Evolutionary Algorithm (EA) [4] which is used to guide the search as it was done by Adrian Thompson when he employed an EA to search for a tone discriminator functionality in a Field Programmable Gate Array (FPGA) chip [31]. He showed that evolution was not only successful in finding the desired functionality, it did so by using the physical properties of the silicon substrate of a reconfigurable chip.

Since it was termed as EIM [20], a number of materials have been used as computing substrates from liquid crystals $[8,9]$ to recent experiments with carbon nanotubes and gold particles within NASCENCE project $[3,15]$. A range of benchmarking computational tasks has been successfully solved which concept-proofed that EIM approach can be used for solving computational tasks.

Figure 1 shows a block diagram of the main parts under EIM. On one side, there is a material which is used as a computational substrate and whose physical properties are used to perform computation. The material is interfaced to a digital computer which runs an EA. The interface is needed for translation of evolutionary steps digitally encoded in a

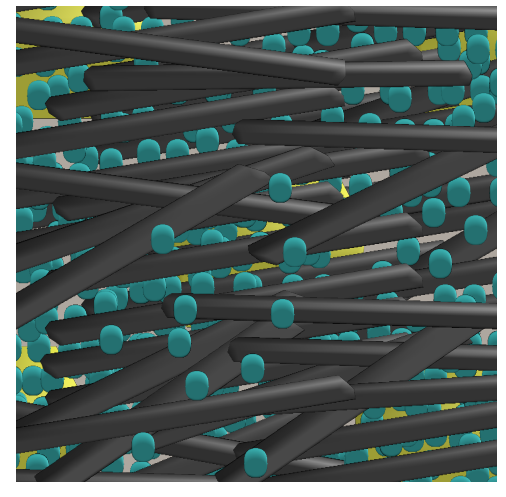

Figure 2: SWCNT-PBMA nanocomposite, a sketch.

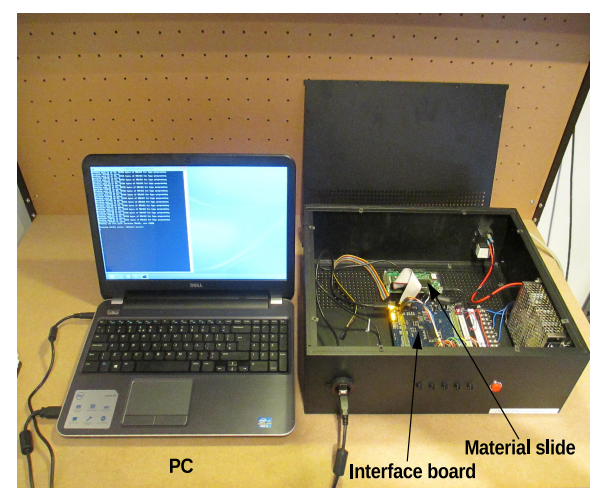

Figure 3: An EIM system, lab setup.

computer to the analogue world of the material physics used for computation. Also, the translation is needed in opposite direction so that the response of the material is interpreted by a digital computer and assessed for fitness within an evolutionary run.

\subsection{Materials used for EIM in NASCENCE}

Figure 3 shows the experimental setup for EIM in our lab. The interface board is called Mecobo [17] and it was developed within the group by Lykkebø for this purpose. Different materials have been investigated. An often used example is nanocomposite based on single-walled carbon nanotubes (SWCNT) dispersed in polymer, Poly-Butyl Methacrylate

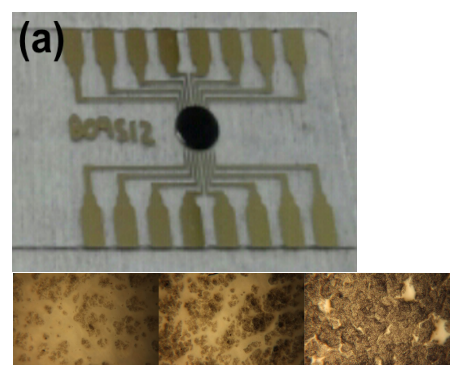

Figure 4: A slide (a) and microscopic view of samples with various CNT concentrations. 
(PBMA) as sketched in Figure 2. Another example of SWCNT based material used for EIM in NASCENCE is SWCNTs mixed with liquid crystals (LC) $[18,32]$. Figure $4^{1}$ shows a blob of the material dispersed on a glass slide over gold electrodes. The slide is connected to the Mecobo board via edge connector.

The main property of the material blob which is used for computations is its conductivity. More precisely it is the change of conductivity that is manipulated for computations. Conductivity of the blob is based on the SWCNT conductivity since polymer molecules are electrically isolators. Bundles of SWCNTs form percolation paths along which the current flows. The current flow is a consequence of the change in the electric field in which the blob of the material is placed. The current and subsequently the conductivity can be changed either by changing the electric field by changing the voltages on the gold electrodes or by changing the position of SWCNTs. The voltages on the electrodes are referred to as configuration signals because of the fact that they determine the electric field and the nanocomposite conductivity. In SWCNT-PBMA nanocomposites, positions of SWCNTs are fixed by polymer molecules and remain such during experiments so that only the change of the electric field can change the conductivity, while both mechanisms are present in SWCNT-LC solutions due to mobility of SWCNTs. The current flow is possible if the paths percolate [30] so that the electrons have uninterrupted path to form a current flow. It is important that the SWCNT concentration in the nanocomposite is such that the percolation threshold is reached and the experiments conducted within NASCENCE have shown that experimental results comply with the theoretical findings [15].

\subsection{Conduction mechanisms in CNTs}

CNTs can electrically be metallic or semiconducting dependent on the chirality. Typically, $\frac{1}{3}$ of the produced CNTs is metallic and remaining $\frac{2}{3}$ semiconducting and such is the case for SWCNTs in our samples. Due to CNT geometrical properties, i.e., the fact that their length is several orders of magnitude larger than their diameter, they are considered one dimensional which electrically makes them behave like nanowires [5]. This leads to a quantized conductivity of electrons along the tube. The conductivity can be ballistic or diffusive. The latter case assumes certain amount of electron scattering due to impurities, lattice defects or phonon scattering. The third mechanism of electron conduction is quantum tunneling. Electrons moving along the nanotubes behave as quantum particles and therefore follow quantum laws a consequence of which is tunelling, i.e., the fact that electrons may appear behind the energy barrier which would be too high to pass if they obeyed just laws of classical mechanics. It is worth noting that quantum effects become more prominent as the temperature lowers.

Bundles of CNTs show more metallic properties [14]. Such behaviour is to be expected since there are more conduction paths in a bundle. However, the conductivity of the CNT bundle shows significant sensitivity to temperature variations.

\footnotetext{
${ }^{1}$ source: NASCENCE internal communication provided by University of Durham
}

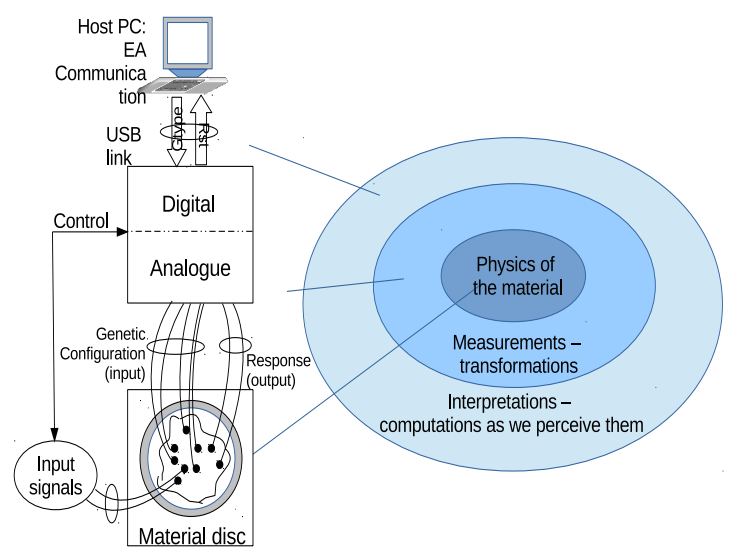

Figure 5: Conceptual domains of the computing system.

\section{COMPUTING WITHIN THE FRAMEWORK OF SYSTEM THEORY}

In the presented example in Section 2, computations are the result of the transformations of the material, transformations being the changes of the electric field in which the material is placed. However, it is the whole system which contributes to achieving computations: the material, computer which runs the EA and the way it interprets signals from the interface board, the interface board and the way it transforms signals between digital and analogue worlds. Therefore, to address computations under EIM scenario, it might be useful to speak of the computing system which performs computations. We acknowledge that the computation happens in the material but due to nanoscale and even quantum nature of the physical transformations within the material, what is captured by our instruments or passed to the digital computer by the interface board might be at best just a good approximation of the true physicality of computations in the material.

Let us take a look at the system which computes in the sense described above. Then, computations can be said to be transformations of a system so that the system input(s) and output(s) are related in some functional way. This functional relation can be captured by a simple formula:

$$
y=F(x)
$$

where $x$ and $y$ correspond to an input and output of the system, respectively, and, in general, they are considered to be multidimensional and represented by vectors.

A framework for explaining and analysing computing or control systems was developed in the middle of the last century [1] by William Ross Ashby, a classical cyberneticist and a member of the Ratio Club [12]. System theory framework assumes that the system state is described by a set of variables. When variables change, the system changes its state in a state space. Further, the system state space is parameterised - for certain value of a parameter, the system moves along one trajectory through its state space as its variables change; for another value of the parameter, the system follows different trajectory for the same changes of its variables. 


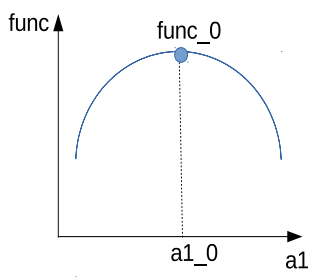

a)

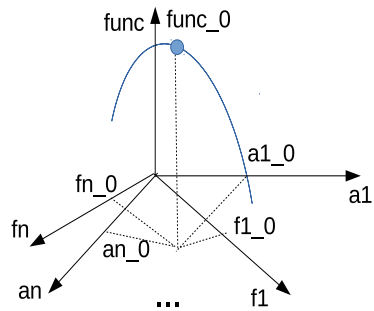

c)

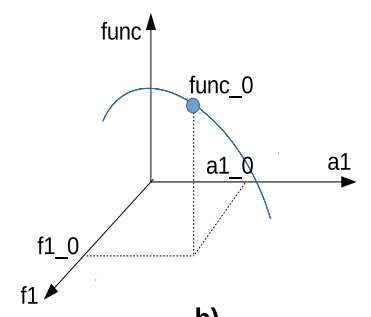

b)

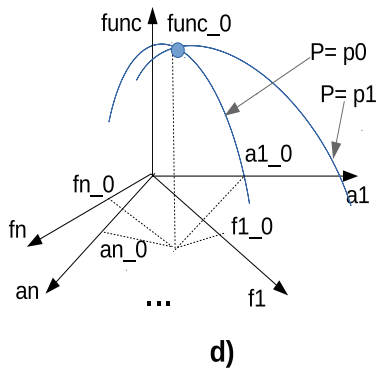

Figure 6: CNT computing system within system theory framework, see text for explanation.

The areas of the state space where the system performes desired functionality were termed ultrastable states by Ashby. In general, there would be more than one ultrastable state for a given system and a given functionality. This was especially exploited in adaptation mechanisms, extensively investigated by Ashby. The main findings in this regard were that if some of the system variables change so that the system moves away from its ultrastable state thereby losing desired functionality, it is possible to change variables so as to lead the system either back to the previous ultrastable state or to the new ultrastable state in which the desired functionality is recovered. This is possible to achieve also by changing the value of the parameter for a parameterised system space [1].

\subsection{Computations within nanomaterials under EIM}

In order to explain computations in the system under EIM scenario within the Ashby's system theory, let us clarify that when system variables are mentioned, although we understand that the true variables which define material behaviour should be addressed at nanoscale and even quantum level, we shall limit ourselves to the values measurable by our instruments, i.e., voltages and currents captured during the EIM experiments. In other words, the variables of the system will be based on the signals and parameters used to configure computing substrate which are measurable by our instruments and referred to as a domain of measurements in Figure 5. The voltages and the set of properties which define them, (amplitude, frequency, phase) can be represented with:

$$
v_{i}=a_{i} \cdot \operatorname{func}_{p}\left(f_{i}, \phi_{i}\right)
$$

where $v_{i}$ is voltage on the $i$-th electrode, $a_{i}$ the amplitude, $f u n c_{p}$ some periodic function, $f_{i}$ frequency of the function func $_{p}$ and, finally, $\phi_{i}$ the phase of the voltage, all referring to the $i$-th electrode. The symbols are left lower case to remind that all of these values can be time varying.
Let us now consider an example in which for a system to perform functionality $f u n c_{-} 0$, for the input $x_{-} 0$, an output value $y_{-} 0$ is desired see Figure $6 a$ ). The variables on each of the axes belong to the conceptual domain of measurements see Figure 5 and are assumed to be scalars for simplicity. When different configuration voltages are applied to the material, they change the system variables so that it passes through various states in the state space along some trajectory. Further, let us assume that only one electrode is used for configuration voltage and only one voltage parameter is changed, amplitude, for example. By changing the amplitude along the $a_{-} 1$ axis different functionalities will be performed by the system. EIM would then search through the space until func_0 point is reached. If also the frequency of the voltage $v_{-} 1$ is changed, then the state space could be searched along two axes as shown in Figure $6 b$ ). And even further, if more than one electrode is used for configuring the material, then, in general, the space would look something like in Figure $6 c$ ) and would be searchable along high number of axes, the limitation being only the physical number of electrodes in the system.

Another case to consider is the change of various parameters in the description of the system state. In Figure 6 this is illustrated for case $d$ ) where one trajectory represents system behaviour for parameter value $P=p 0$ and another for parameter value $P=p 1$. Let us take temperature for example. Under one temperature, the system will give one response. If the temperature changes, it affects the computing substrate so that a different trajectory in the state space will be passed. For the case illustrated in Figure $6 d$ ) this means that the point func_0 could be reached for various parameter $\mathrm{P}$ values.

This leads to an interesting observation. If the state space is represented in such a way so that the parameter, e.g., temperature is a variable which can be controllably changed during the search for a desired solution, the same system with the same computational capabilities can be described with a bigger choice of variables. In other words, there are more spaces in which the system can be explored, which one is chosen depends on the interpretation of the physical variables at hand and on representations of the physical properties of the computing substrate. For example, if temperature is used as a variable in the description of the system state space, we can talk about computing with temperatures in a sense we speak of computing with voltages in, for example, a Travelling Salesman Problem solved by CNT blobs [3]. Similar findings were achieved in reconfigurable hardware known as polymorphic electronics [23, 28]. For certain value of a parameter, in the cases considered it was either a power voltage or an environmental parameter - temperature, the evolved circuit performed one functionality, e.g., a logic AND gate, while for another value of a parameter, the functionality exhibited by the same circuit was different, e.g., logic OR gate.

\subsection{The size of the space of the blob of CNTs}

When an EIM computing system is described within the system theory framework, variables used to describe the state of the system are those which belong to the conceptual domain of measurements. The state of the system and the material is described by the voltages brought to the electrodes on the material slide. However, the physics of the nanocomposites under investigation is much richer than 
what can be represented with given number of electrodes and the voltages on them. As described in Section 2.2, for a special case of SWCNT nanocomposites, the flow of electrons is achieved by several mechanisms.

In order to truly address the size of the state space of the computing substrate, a careful look into its physics must be taken and for electrons in SWCNTs it is the world of quantum physics. As there is "only one reality to be described, which happens to have two different aspects" 2 , it is useful to understand how the measured values are related to the values which are the most accurate description of the system, i.e., the quantum nature of the electrons. The "one reality" and its descriptions are nicely addressed in [19]. On one side, Maxwell's equations [25], which are the basis for classical electrodynamics, establish relations between the electric and the magnetic field, and the charge and the currents pertaining to the same electromagnetic field. But, we may ask what these basic notions of a field are. Analysis shows that it is just a manifestation of the effect an electric charge has on another electric charge if found within certain distance from the former. If the charge is static, its effects will be perceived as electrostatic field, if dynamic, i.e., the charge in motion, it will be perceived as a magnetic field, to simplify the explanation.

In [19], the same laws are revised and put into the framework of the quantum nature of electrons which make up for the current flow. Equivalent description of the electrodynamics as described by Maxwell's equations and circuit equations, is given by the wave functions of the moving electrons, i.e., the phase and the propagation vector of these wave functions. Because electrodynamics is exactly that electrons in motion - the nature of electrons is best captured by the laws of quantum physics and the wave functions which describe them. Such view leads us to three descriptions of the electrodynamic system: by circuit elements, i.e., voltage and current, by four-potential and as a collective property of the electrons described by their wave functions. Detailed derivation of how each refers to the others is provided in [19] and equations are omitted at this place. What is important to note is that those three descriptions are equivalent and equally good at describing the behaviour of the electromagnetic system. It is also worth noticing that at the very bottom, in its true essence, the movement of the electrons as quantum particles and the emergent property observed by us as electric current, can be explained as a collective property of electrons - the accumulation of the phase of their wave functions corresponds to the current.

\section{FROM TOP TO THE BOTTOM AND BACK}

The emergence of higher hierarchical levels is one of the central themes in many disciplines. Hierarchies are a powerful tool in the hands of the architect of complexity [27,29]. Rising from the dynamics of the units at low levels, hierarchies have attracted a lot of attention in an attempt to explain them or give them a suitable mathematical framework $[7,11,16,26]$, and yet, there are many open questions around this topic, for example, what defines a new, higher level: is it a structure, or new functionality, or novel information contained in the higher level dynamics. One thing is certain, though, and that is the dynamics of the units at a lower level gives rise to novel properties at a higher level.

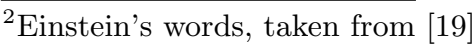

We claim that in the examples of the computing systems investigated under the EIM scenario in our experiments the property of novelty arising from the dynamics of the units at a low level is beautifully represented. At the very bottom there are quantum laws which guide electrons through the mesh of bundles of carbon nanotubes. At the very bottom, electrons behave like quantum particles decribed by wave functions. Electromagnetic field in the nanocomposite is a manifestation of a collective property of electrons in motion which are described by wave functions [19]. The collective electrodynamics, collective property of electrons in the computing substrate as described by ensembles of wave functions and their phases gives rise to currents and potential drops and correspondingly manifests electric and magnetic fields in the material which are measurable by our instruments.

The question then arises naturally: if we look into the space of a computing substrate at this low level, manipulate the system by changing the variables which affect state at this level, what can be gained? Can the gain be in having less variables to control? Or is the gain in a simpler way in which they are manipulated at this low level? These questions open not only additional dimensions in the state space, they also open new research directions for the unconventional computing systems under our investigation.

\section{CONCLUSION}

In this short paper an attempt was made to explain computations which happen in nanomaterials under EIM scenario. As a special case SWCNT based nanocomposites are considered where computations are the result of a changing conductivity of the material due to the changes of the electric field. Further, as computations arise as a result of the material physics, we have addressed the mechanisms of electron transport as well as laws which guide them at this low level from the perspective of quantum mechanics. In this light, the considered computing system represents a manifestation of dynamical hierarchies where electromagnetic field arises as a collective property of wave functions which describe electrons at the quantum level. The perceived computation is the result of changes at this low, i.e., quantum level. On the other hand, manipulation of the material and observation of computations happen at a macroscopic level where electromagnetic properties are measurable. Computations are perceived at a higher hierarchical level than the true physics which guides the changes in the material. However, our analysis of true physical transformations shows that manipulation of the state of the computing substrate can be done at even lower - quantum level. Even more, when the EIM system is set within the framework of system theory, another possibility for achieving computations becomes clear, the one based on manipulation by parameters such as temperature.

To conclude, the presented analysis not only helps understand the processes in the investigated computing EIM systems, it also helps identify future research directions. One is particularly challenging which suggests manipulation of quantum properties of electrons in the material so that the emerging electromagnetic properties can be used for computations. This may sound far fetched, especially when today's state of quantum computers is considered, but it might be exactly this way of manipulation that will bring CNT based nanocomposites and EIM forward and closer to the semiconductor supremacy for computing machines. 


\section{ACKNOWLEDGMENTS}

The research presented in this paper has been funded within EC 7FP under grant agreement no. 317662

\section{REFERENCES}

[1] W. R. Ashby. Design for a Brain, the origin of adaptive behaviour. Chapman \& Hall Ltd., 1960.

[2] H. Broersma, F. Gomez, J. Miller, M. Petty, and G. Tufte. Nascence project: Nanoscale engineering for novel computation using evolution. International Journal of Unconventional Computing, 8(4):313-317, 2012.

[3] K. Clegg, J. Miller, K. Massey, and M. Petty. Travelling salesman problem solved Śin materioŠ by evolved carbon nanotube device. In

T. Bartz-Beielstein, J. Branke, B. Filipic, and J. Smith, editors, Parallel Problem Solving from Nature Ü PPSN XIII, volume 8672 of Lecture Notes in Computer Science, pages 692-701. Springer International Publishing, 2014.

[4] K. de Jong. Evolutionary Computation, a Unified Approach. The MIT Press, 2006.

[5] C. Dekker. Carbon nanotubes as molecular quantum wires. Physics Today, 52(5):22-28, 1999.

[6] D. Deutsch. The Beginning of Infinity. Viking Adult, 2011.

[7] A. Dorin and J. McCormack. Self-assembling dynamical hierarchies. In Proceedings of the eighth international conference on Artificial life, pages 423-428, Cambridge, MA, USA, 2003. MIT Press.

[8] S. Harding and J. Miller. Evolution in materio: a tone discriminator in liquid crystal. In Evolutionary Computation, 2004. CEC2004. Congress on, volume 2, pages 1800-1807 Vol.2, 2004.

[9] S. Harding and J. F. Miller. Evolution in materio : A real-time robot controller in liquid crystal. In J. Lohn, D. Gwaltney, G. Hornby, R. Zebulum, D. Keymeulen, and A. Stoica, editors, Proceedings of the 2005 NASA/DoD Conference on Evolvable Hardware, pages 229-238, Washington, DC, USA, 29 June-1 July 2005. IEEE Press.

[10] S. Harding, J. F. Miller, and E. A. Rietman. Evolution in materio: Exploiting the physics of materials for computation. Int $J$ of Unconventional Computing, pages 155-194, 2008.

[11] e. Howard H. Pattee. Hierarchy Theory. George Braziller, 1973.

[12] P. Husbands and O. Holland. The Ratio Club: A hub of British cybernetics. In P. Husbands, O. Holland, and M. Wheeler, editors, The Mechanical Mind in History, pages 91-148. The MIT Press, 2008.

[13] P. Husbands, O. Holland, and M. Wheeler. The Mechanical Mind in History. The MIT Press, 2008.

[14] A. B. Kaiser. Electronic transport properties of conducting polymers and carbon nanotubes. Reports on Progress in Physics, 64(1):1, 2001.

[15] A. Kotsialos, K. Massey, F. Qaiser, D. Zeze, C. Pearson, and M. Petty. Logic gate and circuit training on randomly dispersed carbon nanotubes. International Journal of Unconventional Computing, 5(6):473-497, 2014.
[16] T. Lenaerts, D. Chu, and R. Watson. Dynamical hierarchies. In Artificial Life, volume 11, pages 403-405, 2005.

[17] O. Lykkebø, S. Harding, G. Tufte, and J. Miller. Mecobo: A hardware and software platform for in materio evolution. In O. H. Ibarra, L. Kari, and S. Kopecki, editors, Unconventional Computation and Natural Computation, Lecture Notes in Computer Science, pages 267-279. Springer International Publishing, 2014.

[18] K. Massey, D. Volpati, F. Qaiser, A. Kotsialos, C. Pearson, D. Zeze, and M. Petty. Alignment of liquid crystal/carbon nanotube dispersions for application in unconventional computing. AIP Conference Proceedings, 1648(1):-, 2015.

[19] C. A. Mead. Collective Electrodynamics: Quantum Foundations of Electromagnetism. MIT Press, 2000.

[20] J. Miller and K. Downing. Evolution in materio: looking beyond the silicon box. In Proceedings of the NASA/DoD Conference on Evolvable Hardware, pages 167-176. NASA/DoD, July 2002.

[21] J. Miller, S. Harding, and G. Tufte. Evolution-in-materio: evolving computation in materials. Evolutionary Intelligence, 7(1):49-67, 2014.

[22] NASCENCE. official web site. http://www.nascence.eu.

[23] J. N. T. R. NPO-21213. Polymorphic electronics - a novel type of circuits with multiple functionality. In NASA Tech Briefs, NANOTECH BRIEF Vol.28, No.4, April 2004.

[24] G. Pask. Physical analogues to the growth of a concept. In Mechanisation of Thought Process, symposium proceedings, pages 877-923, 1958.

[25] E. M. Purcell and D. J. Morin. Electricity and Magnetism. Cambridge University Press, 2013.

[26] S. Rasmussen, N. Baas, B. Mayer, M. Nilsson, and M. Olesen. Ansatz for dynamical hierarchies. In Artificial Life, pages 329-353. Massachusetts Institute of Technology, 2001.

[27] S. N. Salthe. Evolving Hierarchical Systems. Columbia University Press, 1985.

[28] L. Sekanina. Design methods for polymorphic digital circuits. In Proceedings of 8th IEEE Design and Diagnostic of Electronic Circuits and Systems Workshop, April 2005.

[29] H. Simon. The architecture of complexity. In Proceedings of the American Philosophical Society, volume 106, pages 467-482, 1962.

[30] D. Stauffer and A. Aharony. Introduction to Percolation Theory. CRC Press, 1994.

[31] A. Thompson. Hardware Evolution: Automatic design of electronic circuits in reconfigurable hardware by artificial evolution. Springer, 1996.

[32] D. Volpati, K. Massey, D. W. Johnson, A. Kotsialos, F. Qaiser, C. Pearson, K. S. Coleman, G. Tiburzi, D. A. Zeze, and M. C. Petty. Exploring the alignment of carbon nanotubes dispersed in a liquid crystal matrix using coplanar electrodes. Journal of Applied Physics, 117(12):-, 2015. 